\title{
THE GEHRING LEMMA: DIMENSION FREE ESTIMATES
}

\author{
JAN KRISTENSEN AND BIANCA STROFFOLINI
}

\begin{abstract}
We present a dimension free estimate for the exponent of higher integrability in Gehring's Lemma for an inhomogeneous weak reverse Hölder inequality.

Dedicated to Carlo Sbordone on the occasion of his 70th birthday.
\end{abstract}

\section{INTRODUCTION AND STATEMENT OF RESULT}

It is well-known that reverse Hölder inequalities are self-improving in the sense that if for some cube $\Omega \subset \mathbb{R}^{n}$, exponent $p>1$ and nonnegative integrable function $f: \Omega \rightarrow \mathbb{R}$ we have that

$$
f_{Q} f^{p} \mathrm{~d} x \leq A_{p}\left(f_{Q} f \mathrm{~d} x\right)^{p}
$$

holds for all subcubes $Q \subset \Omega$ with a constant $A_{p}$ independent of the cube $Q$, then there exist an exponent $q>p$ and a constant $A_{q}$, both depending on $n, p$ and $A_{p}$, such that

$$
f_{Q} f^{q} \mathrm{~d} x \leq A_{q}\left(f_{Q} f \mathrm{~d} x\right)^{q}
$$

holds for all subcubes $Q \subset \Omega$. The result is known as Gehring's Lemma [10]. The interest in reverse Hölder inequalities can be traced back at the least to the work of Muckenhoupt [29] and the theory of $\mathrm{L}^{p}$ weights (see also [3, 4, 9]). However, Gehring's contribution was in the direction of $\mathrm{L}^{p}$ theory of quasiconformal mappings. For quasiregular mappings and more generally nonlinear PDEs, weak reverse Hölder inequalities appear naturally in connection with Caccioppoli type estimates. A weak reverse Hölder inequality often takes the form

$$
f_{Q} f^{p} \mathrm{~d} x \leq A_{p}\left(f_{2 Q} f \mathrm{~d} x\right)^{p}
$$

where we now integrate over the double cube $2 Q$ on the right-hand side. That validity of these inequalities and their inhomogeneous variants also self-improve was established through the works of Elcrat and Meyers [27], Giaquinta and Modica [11] and Stredulinsky [31]. Since then such inequalities have been widely studied in many different situations, see for instance [12, 14] for more details and background. More recently, there has been some interest in obtaining a better control of the constants that appear in the self-improved inequality. In particular it has been shown that dimension free integrability improvement can be obtained from a strong reverse Hölder inequality in the sense that the exponent $q>p$ above can be taken independent of the dimension $n$ in such cases. Here we use a terminology that is inspired by Kinnunen [16] who defined a notion of strong Muckenhoupt weights, and thus a strong reverse Hölder inequality is an inequality of the form

$$
f_{R} f^{p} \mathrm{~d} x \leq A_{p}\left(f_{R} f \mathrm{~d} x\right)^{p}
$$

where now $R$ is any rectangle in $\mathbb{R}^{n}$ (no bound on the ratios between side-lengths). See also [6, 21, $24,30,32]$ for further interesting results in this direction. In the present note we show that dimension free integrability improvement follows from an inhomogeneous weak reverse Hölder inequality on rectangles $R$ whose side lengths have ratios between $1 / 2$ and 2 (we call them admissible rectangles): 
Theorem 1.1. Let $\Omega$ be an open subset of $\mathbb{R}^{n}, p \in(1, \infty), K \geq 1$ and $f, h \in \mathrm{L}^{p}(\Omega)$ be nonnegative. Assume that

$$
\left(f_{R} f^{p} \mathrm{~d} x\right)^{\frac{1}{p}} \leq K f_{2 R} f \mathrm{~d} x+\left(f_{2 R} h^{p} \mathrm{~d} x\right)^{\frac{1}{p}}
$$

holds for all admissible rectangles $R$ with $2 R \subset \Omega$. Then for every exponent $q$ satisfying

$$
q \leq p+\frac{p}{2^{p+2} K^{p+1}\left(\frac{p}{p-1}\right)^{2} \mathrm{e}^{2}-1}
$$

we have for cubes $Q$ whose double $2 Q \subset \Omega$ the bound

$$
\left(f_{Q} f^{q} \mathrm{~d} x\right)^{\frac{1}{q}} \leq A\left(f_{2 Q} f^{p} \mathrm{~d} x\right)^{\frac{1}{p}}+B\left(f_{2 Q} h^{q} \mathrm{~d} x\right)^{\frac{1}{q}}
$$

where $A=2^{2 n+1}$ and $B=4^{n+3}\left(\frac{p}{p-1}\right)^{6} K^{3}$ will do.

We emphasize that the main point of our result is the dimension free integrability improvement and that we have not made any attempt at providing the sharp constants. Our proof is modelled on a proof of Iwaniec [13] and relies on having good control over constants in various maximal inequalities. These bounds are established in Section 3 and the proof of Theorem 1.1 is presented in Section 4. The fact that our proof relies mainly on martingale maximal inequalities makes it more elementary than those recorded in the literature.

Among the earlier references on reverse Hölder inequalities and the closely related topic of Muckenhoupt weights we mention $[1,5,28]$ and for a sample of applications and extensions not included in the monographs mentioned above see for instance $[2,8,15,18,19,20]$.

\section{PRELiminaries}

2.1. Basic notation. On $\mathbb{R}^{n}$ we use standard euclidean inner products and the corresponding norms denoted by $x \cdot y$ and $|x|=\sqrt{x \cdot y}$, respectively. When $A$ and $B$ are subsets of $\mathbb{R}^{n}$, then the distance between them is as usual defined by $\operatorname{dist}(A, B)=\inf \{|a-b|: a \in A, b \in B\}$.

We use standard notation for functions and measures as can be found in for instance [22]. In particular, the Lebesgue measure on $\mathbb{R}^{n}$ is denoted by $\mathscr{L}^{n}$ and when $f: A \rightarrow[0, \infty]$ is a nonnegative measurable function defined on a measurable subset $A \subset \mathbb{R}^{n}$ with $\mathscr{L}^{n}(A) \in(0, \infty)$ its integral mean over $A$ is denoted by

$$
f_{A} f(x) \mathrm{d} x=f_{A} f \mathrm{~d} x:=\frac{1}{\mathscr{L}^{n}(A)} \int_{A} f(x) \mathrm{d} x,
$$

where the value $\infty$ as usual is allowed.

2.2. Dyadic cubes and rectangles. By a cube $Q$ in $\mathbb{R}^{n}$ we mean a closed cubic interval, so for some $a \in \mathbb{R}^{n}$ and $s>0, Q=a+\left[-\frac{s}{2}, \frac{s}{2}\right]^{n}=\left[a_{1}-\frac{s}{2}, a_{1}+\frac{s}{2}\right] \times \cdots \times\left[a_{n}-\frac{s}{2}, a_{n}+\frac{s}{2}\right]$. We refer to $a$ as the center of the cube $Q$ and $s$ as its sidelength. For a positive number $\lambda>0$ we write $\lambda Q$ for the dilated cube obtained from $Q$ by dilation with factor $\lambda$ about its center $a$.

For a cube $Q$ in $\mathbb{R}^{n}$ its dyadic subcubes are the cubes obtained by simultaneously bisecting all sides of the cube and its subcubes. We refer to $Q$ itself as the 0 -th generation dyadic subcube, and each of the $2^{n}$ congruent subcubes obtained by bisecting each side of $Q$ as the 1-st generation and so on. The collection of $k$-th generation dyadic subcubes of $Q$ is denoted by $\mathcal{D}_{k}(Q)$ and we record that it consists of $2^{k n}$ nonoverlapping congruent subcubes. Each $k$-th generation dyadic subcube is in turn the nonoverlapping union of the $2^{n}(k+1)$-th generation dyadic subcubes it contains and we refer to these dyadic subcubes as its children and in turn the $k$-th generation subcube as their parent. The family of all dyadic subcubes of $Q$ is denoted by $\mathcal{D}(Q)=\bigcup_{k=0}^{\infty} \mathcal{D}_{k}(Q)$.

By a rectangle $R$ in $\mathbb{R}^{n}$ we mean a cartesian product of $n$ bounded closed intervals $R=\left[a_{1}, b_{1}\right] \times$ $\cdots \times\left[a_{n}, b_{n}\right]$. The center of $R$ is $\left(\left(b_{1}-a_{1}\right) / 2, \ldots,\left(b_{n}-a_{n}\right) / 2\right)$ and for a positive number $\lambda>0$ the dilated rectangle $\lambda R$ is the rectangle obtained by dilating $R$ about its center with factor $\lambda$. 
We shall call a rectangle in $\mathbb{R}^{n}$ for admissible if the ratios of its sidelengths $b_{i}-a_{i}$ all belong to the interval $\left[\frac{1}{2}, 2\right]$ :

$$
\frac{1}{2} \leq \frac{b_{i}-a_{i}}{b_{j}-a_{j}} \leq 2 \text { for } 1 \leq i, j \leq n .
$$

The notion of dyadic rectangles is defined as a natural generalization of that of dyadic cubes. As a starting point we fix an admissible rectangle $Q$ in $\mathbb{R}^{n}$. The 0 -th generation dyadic rectangle is $Q$ itself, and we put $\mathcal{R}_{0}(Q)=\{Q\}$. The first generation dyadic rectangles are the two congruent rectangles, $R^{\prime}, R^{\prime \prime}$, obtained by bisecting the first coordinate side of $Q$, and we put $\mathcal{R}_{1}(Q)=\left\{R^{\prime}, R^{\prime \prime}\right\}$. The second generation dyadic rectangles are the four congruent rectangles that result when we bisect the second coordinate side in $R^{\prime}$ and $R^{\prime \prime}$, and so on. The $k$-th generation dyadic rectangles is denoted by $\mathcal{R}_{k}(Q)$ and the family of all dyadic rectangles is denoted by $\mathcal{R}(Q)=\bigcup_{k=0}^{\infty} \mathcal{R}_{k}(Q)$. We record that $\mathcal{R}_{k}(Q)$ consists of $2^{k}$ nonoverlapping congruent rectangles, each of which is the union of the two $(k+1)$-th generation dyadic rectangles it contains. We refer to these two $(k+1)$-th generation dyadic rectangles as its children and in turn the $k$-th generation dyadic rectangle as their parent. Clearly, the $n$ th generation dyadic rectangles coincide with the 1-st generation dyadic cubes when the starting point $Q$ is a cube.

Lemma 2.1. Let $Q$ be an admissible rectangle in $\mathbb{R}^{n}$ with center $a \in \mathbb{R}^{n}$ and let $k \in \mathbb{N}_{0}$. Then for each $R \in \mathcal{R}_{k}(Q)$ we have $2 R \in \mathcal{R}_{k}(2 Q)$ and

$$
2 R=\bigcup\left\{S \in \mathcal{R}_{k+n}(2 Q): S \subset 2 R\right\}
$$

a nonoverlapping union of $2^{n}$ dyadic $(k+n)$-th generation subrectangles $R_{1}, \ldots, R_{2^{n}}$ that each satisfies $R_{j} \cap R \in \mathcal{R}_{k+n}(Q)$ and $2^{n} \mathscr{L}^{n}\left(R_{j} \cap R\right)=\mathscr{L}^{n}\left(R_{j}\right)$.

Proof. Using a coordinate transformation we can assume that $Q=[-1 / 2,1 / 2]^{n}$. Since each dyadic rectangle is a nonoverlapping union of the maximal dyadic cubes it contains we can without loss in generality also assume that $R$ is a dyadic cube: $R=2^{-k}(z+Q)$ for some $z \in \mathbb{Z}^{n}$. But then $2 R=$ $2^{-k}(z+2 Q) \in \mathcal{R}_{k}(2 Q)$ and so $2 R$ is the nonoverlapping union of the $2^{n}$ dyadic $(n+k)$-th generation subrectangles, $R_{1}, \ldots, R_{2^{n}}$, of $2 Q$ that it contains. It is geometrically clear that $R_{j} \cap R \in \mathcal{R}_{k+n}(Q)$ and that $2^{n} \mathscr{L}^{n}\left(R_{j} \cap R\right)=\mathscr{L}^{n}\left(R_{j}\right)$, so the proof is complete.

\section{MAXimal inequalities}

For a cube $Q$ in $\mathbb{R}^{n}$ we define for each nonnegative $f \in \mathrm{L}^{1}(Q)$ its maximal function corresponding to the family $\mathcal{R}(Q)$ of dyadic rectangles as

$$
M[f](x)=M_{\mathcal{R}(Q)}[f](x):=\sup \left\{f_{R} f \mathrm{~d} y: R \in \mathcal{R}(Q) \text { and } x \in R\right\} \quad(x \in Q)
$$

Clearly, $M[f]: Q \rightarrow[0, \infty]$ is a measurable function and we denote its distribution function by

$$
\lambda(t):=\mathscr{L}^{n}(\{x \in Q: M[f]>t\}) \quad(t \geq 0)
$$

From the martingale convergence theorem follows that $M[f] \geq f$ almost everywhere (see for instance [7, Chap. XI, Sect. 14]). We then have the following Doob type maximal-martingale inequalities:

Proposition 3.1. For all $t>f_{Q}$ we have

$$
\frac{1}{2} \int_{\{x: M[f]>t\}} f \mathrm{~d} x \leq t \lambda(t) \leq \int_{\{x: M[f]>t\}} f \mathrm{~d} x,
$$

and consequently for each $p \in(1, \infty)$ the integral bounds

$$
\left(\frac{p^{\prime}}{2} f_{Q} f^{p} \mathrm{~d} x\right)^{\frac{1}{p}}-\left(\frac{p^{\prime}}{2}\right)^{\frac{1}{p}} f_{Q} \leq\left(f_{Q} M[f]^{p} \mathrm{~d} x\right)^{\frac{1}{p}} \leq p^{\prime}\left(f_{Q} f^{p} \mathrm{~d} x\right)^{\frac{1}{p}},
$$

where we recall the notation $p^{\prime}=\frac{p}{p-1}$ for the Hölder conjugate exponent. 
Remark 3.2. While the upper integral bound in (3.2) is sharp, it is in fact possible to slightly refine both bounds appearing in (3.2). For instance, when $p=2$ the reader can readily check from the proof given below that (3.1) actually yields

$$
f_{Q} f^{2} \mathrm{~d} x \leq f_{Q} M[f]^{2} \mathrm{~d} x \leq f_{Q} f^{2} \mathrm{~d} x+f_{Q}\left|f-f_{Q}\right|^{2} \mathrm{~d} x .
$$

The corresponding estimates for $p \neq 2$ are more involved, and as we do not require them here we will not pursue them further. Anyway, using more sophisticated methods a whole array of such refined bounds have been obtained in [23, 25].

Proof. For the proof of (3.1) we fix $t>f_{Q}$ and put $F=\{x \in Q: M[f]>t\}$. Consider the family $\mathcal{F}$ of dyadic rectangles $R \in \mathcal{R}$ satisfying

$$
f_{R} f \mathrm{~d} x>t
$$

Each $R \in \mathcal{F}$ is contained in a unique maximal $\bar{R} \in \mathcal{F}$. Denote by $R_{j}, j \in J$, the collection of such maximal dyadic rectangles and note that $F=\bigcup_{j \in J} R_{j}$, as a non-overlapping union. Since $t>f_{Q}$ we have $R_{j} \neq Q$ and the dyadic parent $\bar{R}_{j} \in \mathcal{R}$ of $R_{j}$ satisfies $f_{\bar{R}_{j}} \leq t$. Since $\bar{R}_{j}$ has two children in $\mathcal{R}$ we deduce that $f_{R_{j}} \leq 2 t$. We can now conclude with (3.1) as follows:

$$
t \mathscr{L}^{n}(F)=\sum_{j \in J} t \mathscr{L}^{n}\left(R_{j}\right)\left\{\begin{array}{l}
<\sum_{j \in J} \int_{R_{j}} f \mathrm{~d} x=\int_{F} f \mathrm{~d} x \\
\geq \sum_{j \in J} \frac{1}{2} \int_{R_{j}} f \mathrm{~d} x=\frac{1}{2} \int_{F} f \mathrm{~d} x .
\end{array}\right.
$$

The derivation of (3.2) is routine: writing $\|\cdot\|_{q}$ for the $\mathrm{L}^{q}$ norm on $Q$ and expressing the integral of $M[f]$ in terms of its distribution function we get

$$
\begin{aligned}
\|M[f]\|_{p}^{p} & =p \int_{0}^{\infty} t^{p-1} \lambda(t) \mathrm{d} t \\
& \stackrel{(3.1)}{\leq} p \int_{0}^{f_{Q}} t^{p-1} \lambda(t) \mathrm{d} t+p \int_{f_{Q}}^{\infty} t^{p-2} \int_{\{x: M[f]>t\}} f \mathrm{~d} x \mathrm{~d} t \\
& \leq\left(f_{Q}\right)^{p} \mathscr{L}^{n}(Q)+p^{\prime} \int_{Q}\left((M[f])^{p-1}-\left(f_{Q}\right)^{p-1}\right) f \mathrm{~d} x \\
& \stackrel{\text { Hölder }}{\leq} p^{\prime}\|M[f]\|_{p}^{p-1}\|f\|_{p}-\frac{1}{p-1}\left(f_{Q}\right)^{p} \mathscr{L}^{n}(Q) .
\end{aligned}
$$

The upper bound in (3.2) follows. The derivation of the lower bound is similar, where instead of Hölder's inequality we simply use that $M[f] \geq f$ a.e. whereby we arrive at

$$
\begin{aligned}
\|M[f]\|_{p}^{p} & \geq \frac{p^{\prime}}{2}\|f\|_{p}^{p}+\frac{p-2}{2(p-1)}\left(f_{Q}\right)^{p} \mathscr{L}^{n}(Q) \\
& \geq \frac{p^{\prime}}{2}\left(\|f\|_{p}^{p}-\left(f_{Q}\right)^{p} \mathscr{L}^{n}(Q)\right)
\end{aligned}
$$

and the lower bound follows easily from this.

Remark 3.3. The usual dyadic maximal function admits slightly worse lower integral bounds that result from each dyadic cube having $2^{n}$ children instead of just 2 . Indeed the changes to the above are minor and we simply must replace the factor $\frac{1}{2}$ in the lower bounds of (3.1), (3.2) by $\frac{1}{2^{n}}$. As we seek to avoid a dependence on the dimension this small change is crucial here.

In order to deal with the weak reverse Hölder inequalities where we integrate over the double rectangle on the right-hand side we require a variant of the upper bound in (3.2). This is addressed in the following 
Proposition 3.4. Let $Q$ be a cube in $\mathbb{R}^{n}$ and $2 Q$ its double. We now consider the maximal function corresponding to the family $\mathcal{R}(2 Q)$ :

$$
M[f](x)=M_{\mathcal{R}(2 Q)}[f](x):=\sup \left\{f_{R} f \mathrm{~d} y: R \in \mathcal{R}(2 Q) \text { and } x \in R\right\} \quad(x \in 2 Q)
$$

where $f \in \mathrm{L}^{1}(2 Q)$ is nonnegative. Put

$$
\lambda(t):=\mathscr{L}^{n}(\{x \in Q: M[f](x)>t\}) \quad(t \geq 0),
$$

where we emphasize that this is the distribution function of $M[f]$ on the cube $Q$ and not on $2 Q$. Then for

$$
t>m:=\max \left\{f_{R} f \mathrm{~d} y: R \in \bigcup_{k=0}^{2 n-1} \mathcal{R}_{k}(2 Q)\right\}
$$

we have

$$
\lambda(t) \leq \frac{1}{t} \int_{\{x \in Q: M[f]>t\}} f \mathrm{~d} x
$$

and consequently for each $p \in(1, \infty)$,

$$
\left(f_{Q} M[f]^{p} \mathrm{~d} x\right)^{\frac{1}{p}} \leq p^{\prime}\left(f_{Q} f^{p} \mathrm{~d} x\right)^{\frac{1}{p}}+m .
$$

Proof. We use Lemma 2.1 to negotiate between the dyadic rectangles with respect to $Q$ and $2 Q$, but proceed otherwise as above. Fix $t>m$ and put $F=\{x \in Q: M[f]>t\}$. We select the maximal rectangles in the family

$$
\mathcal{F}=\left\{R \in \mathcal{R}(2 Q): f_{R} f \mathrm{~d} x>t\right\} .
$$

If $R \in \mathcal{F}$, then necessarily $R \in \mathcal{R}_{k}(2 Q)$ for some $k \geq 2 n$ since $t>m$. But from Lemma 2.1 we have in particular that $Q=\bigcup\left\{T \in \mathcal{R}_{2 n}(2 Q): T \subset Q\right\}$ and so if $\mathscr{L}^{n}(R \cap Q)>0$, then $R \subset Q$. The maximal rectangles in $\mathcal{F}$ therefore fall into two groups, those contained in $Q$ and those contained in the complement $(2 Q) \backslash \operatorname{int} Q$. Denote the former by $R_{j}, j \in J$. Then $F=\bigcup_{j \in J} R_{j}$ as nonoverlapping union and estimating as in the previous proof we find

$$
\lambda(t)=\sum_{j \in J} \mathscr{L}^{n}\left(R_{j}\right)<\frac{1}{t} \sum_{j \in J} \int_{R_{j}} f \mathrm{~d} x=\frac{1}{t} \int_{F} f \mathrm{~d} x,
$$

which is (3.4). Now using this bound we find for $p \in(1, \infty)$ that

$$
\begin{aligned}
\int_{Q} M[f]^{p} \mathrm{~d} x & =\left(\int_{0}^{m}+\int_{m}^{\infty}\right) p t^{p-1} \lambda(t) \mathrm{d} t=: I+I I \\
& \stackrel{(3.4)}{\leq} I+\int_{m}^{\infty} p t^{p-2} \int_{\{x \in Q: M[f]>t\}} f \mathrm{~d} x \mathrm{~d} t \\
& =I+p^{\prime} \int_{\{x \in Q: M[f]>m\}}\left(M[f]^{p-1}-m^{p-1}\right) f \mathrm{~d} x \\
& \stackrel{\text { Hölder }}{\leq} I+p^{\prime}\|M[f]\|_{p}^{p-1}\|f\|_{p}
\end{aligned}
$$

where $\|\cdot\|_{p}$ is the $\mathrm{L}^{p}$ norm on $Q$. Note that $\|M[f]\|_{p} \geq I^{\frac{1}{p}}$ and that the function $X \mapsto X^{p}-p^{\prime} X^{p-1} Y$ is increasing for $X \geq Y$. When $Y \geq 0$ we have for $X=I^{\frac{1}{p}}+p^{\prime} Y$ that $Y=\left(X-I^{\frac{1}{p}}\right) / p^{\prime}$ and therefore that $X^{p}-p^{\prime} X^{p-1} Y=I$. Consequently, $X^{p}-p^{\prime} X^{p-1} Y>I$ for $X>I^{\frac{1}{p}}+p^{\prime} Y$ and therefore (3.5) follows if we take $X=\|M[f]\|_{p}, Y=\|f\|_{p}$ and note that $I \leq m^{p} \mathscr{L}^{n}(Q)$. 
Remark 3.5. The significance of (3.5) resides for us in the fact that the two $\mathrm{L}^{p}$ norms are taken over the cube $Q$ and not its double $2 Q$ despite the maximal function being defined with reference to $2 Q$. Integrals over the double cube $2 Q$ appear in $m$.

We require yet another maximal operator in order to efficiently deal with the weak reverse Hölder inequalities. For its definition, fix again a cube $Q$ in $\mathbb{R}^{n}$ and put for nonnegative $f \in \mathrm{L}^{1}(2 Q)$,

$$
\tilde{M}[f](x)=\tilde{M}_{\mathcal{R}(Q)}[f](x):=\sup \left\{f_{2 R} f \mathrm{~d} y: R \in \mathcal{R}(Q) \text { and } x \in R\right\} \quad(x \in Q)
$$

This maximal function is not of martingale type because the generated $\sigma$-algebras of subsets of $2 Q$, $\sigma\left(\left\{2 R: R \in \mathcal{R}_{k}(Q)\right\}\right), k \in \mathbb{N}_{0}$, do not form a filtration (see [7] for the terminology). However, as we shall see it is possible to bound its distribution function using the maximal operator $M_{\mathcal{R}(2 Q)}$ considered above and then deduce an $\mathrm{L}^{p}$ bound from Proposition 3.4 that is essentially dimension free.

Proposition 3.6. Let $f \in \mathrm{L}^{1}(2 Q)$ be nonnegative and put

$$
\tilde{\lambda}(t):=\mathscr{L}^{n}(\{x \in Q: \tilde{M}[f](x)>t\}) \quad(t \geq 0)
$$

Then for $t>0$ we have

$$
\tilde{\lambda}(t) \leq \frac{1}{t} \int_{\{x \in Q: \tilde{M}[f]>t\}} M_{\mathcal{R}(2 Q)}[f] \mathrm{d} x
$$

and hence for $p \in(1, \infty)$,

$$
\left(f_{Q} \tilde{M}[f]^{p} \mathrm{~d} x\right)^{\frac{1}{p}} \leq\left(p^{\prime}\right)^{2}\left(f_{Q} f^{p} \mathrm{~d} x\right)^{\frac{1}{p}}+p^{\prime} 2^{2 n} f_{2 Q} f \mathrm{~d} x .
$$

Proof. Let $t>0$ and put $\tilde{F}=\{x \in Q: \tilde{M}[f](x)>t\}$. Consider the family $\tilde{\mathcal{F}}$ of dyadic rectangles $R \in \mathcal{R}(Q)$ satisfying

$$
f_{2 R} f \mathrm{~d} x>t
$$

Let $R_{j}, j \in J$, denote the maximal such rectangles. Then $\tilde{\mathcal{F}}=\bigcup_{j \in J} R_{j}$ as a nonoverlapping union, and according to Lemma 2.1 we may write each double $2 R_{j}$ as a nonoverlapping union:

$$
2 R_{j}=Q_{j, 1} \cup \ldots \cup Q_{j, 2^{n}},
$$

where $Q_{j, i} \in \mathcal{R}(2 Q), Q_{j, i} \cap R_{j} \in \mathcal{R}(Q)$ and $\mathscr{L}^{n}\left(Q_{j, i}\right)=2^{n} \mathscr{L}^{n}\left(Q_{j, i} \cap R_{j}\right)$. Now

$$
\begin{aligned}
\tilde{\lambda}(t) & =\sum_{j \in J} \mathscr{L}^{n}\left(R_{j}\right)<\frac{1}{2^{n} t} \sum_{j \in J} \int_{2_{j}} f \mathrm{~d} x \\
& =\frac{1}{2^{n} t} \sum_{j \in J} \sum_{i=1}^{2^{n}} \mathscr{L}^{n}\left(Q_{j, i}\right) f_{Q_{j, i}} f \mathrm{~d} x \\
& \leq \frac{1}{2^{n} t} \sum_{j \in J} \sum_{i=1}^{2^{n}} \mathscr{L}^{n}\left(Q_{j, i}\right) \inf _{Q_{j, i}} M_{\mathcal{R}(2 Q)}[f] \\
& =\frac{1}{t} \sum_{j \in J} \sum_{i=1}^{2^{n}} \mathscr{L}^{n}\left(Q_{j, i} \cap R_{j}\right) \inf _{Q_{j, i}} M_{\mathcal{R}(2 Q)}[f] \\
& \leq \frac{1}{t} \int_{F} M_{\mathcal{R}(2 Q)}[f] \mathrm{d} x .
\end{aligned}
$$


This is (3.6). For (3.7) we proceed as in the previous proof and denoting by $\|\cdot\|_{p}$ the $\mathrm{L}^{p}$ norm taken over $Q$ we get

$$
\begin{aligned}
& \|\tilde{M}[f]\|_{p}^{p} \quad=\quad \int_{0}^{\infty} p t^{p-1} \tilde{\lambda}(t) \mathrm{d} t \\
& \stackrel{(3.6)}{\leq} \int_{0}^{\infty} p t^{p-2} \int_{\{x \in Q: \tilde{M}[f]>t\}} M_{\mathcal{R}(2 Q)}[f] \mathrm{d} x \mathrm{~d} t \\
& =\quad p^{\prime} \int Q \tilde{M}[f]^{p-1} M_{\mathcal{R}(2 Q)}[f] \mathrm{d} x \\
& \stackrel{\text { Hölder }}{\leq} \quad p^{\prime}\|\tilde{M}[f]\|_{p}^{p-1}\left\|M_{\mathcal{R}(2 Q)}[f]\right\|_{p} \\
& \stackrel{(3.4)}{\leq} p^{\prime}\|\tilde{M}[f]\|_{p}^{p-1}\left(p^{\prime}\|f\|_{p}+m \mathscr{L}^{n}(Q)^{\frac{1}{p}}\right)
\end{aligned}
$$

and (3.7) follows since $m \leq 2^{2 n} f_{2 Q}$.

\section{Proof OF THEOREM 1.1}

We follow the strategy of the maximal function proof as exposed in [13, Section 5], but invoke the maximal inequalities from Section 3. The original proof of Gehring [10] and also its generalizations by Elcrat and Meyers [27], Giaquinta and Modica [11] and Stredulinsky [31] used maximal functions too, but relied on a variant of an inequality for Stieltjes integrals from [10] instead of more precise estimates of the constants in the maximal inequalities.

Proof. It is convenient to proceed in two steps.

Step 1. We reduce to the case where $f, h$ are bounded functions.

Let us emphasize that it is at this the point we must require the weak reverse Hölder inequality (1.1) holds for all admissible rectangles and not merely for dyadic rectangles as defined in Section 2 and used in the later steps of this proof. The reduction is identical to that given in [13, Section 5], but we provide details for the convenience of the reader since our setting is slightly different.

Let $\left(\rho_{t}\right)$ be a standard smooth mollifier:

$$
\rho_{t}(x)=\frac{1}{t^{n}} \rho\left(\frac{x}{t}\right), x \in \mathbb{R}^{n}, t>0,
$$

where $\rho \in \mathrm{C}_{c}^{\infty}\left(\mathbb{R}^{n}\right)$ is nonnegative, supported in $B_{1}(0)$, radial and integrates to 1 . We extend $f, h$ to all of $\mathbb{R}^{n}$ by defining them to be 0 off $\Omega$. Put $f_{t}:=\rho_{t} * f$. Then we find for an admissible rectangle $R \subset \Omega$ satisfying $\operatorname{dist}(2 R, \partial \Omega)>t$, using first the integral form of Minkowski's inequality, then (1.1) and finally Jensen's inequality:

$$
\begin{aligned}
\left(f_{R} f_{t}^{p} \mathrm{~d} x\right)^{\frac{1}{p}} & \leq \int_{\mathbb{R}^{n}} \rho_{t}(y)\left(f_{R} f(x-y)^{p} \mathrm{~d} x\right)^{\frac{1}{p}} \mathrm{~d} y \\
& \leq \int_{\mathbb{R}^{n}} \rho_{t}(y)\left[K f_{2 R} f(x-y) \mathrm{d} x+\left(f_{2 R} h(x-y)^{p} \mathrm{~d} x\right)^{\frac{1}{p}}\right] \mathrm{d} y \\
& \leq K f_{2 R} f_{t} \mathrm{~d} x+\left(f_{2 R} H_{t}^{p} \mathrm{~d} x\right)^{\frac{1}{p}},
\end{aligned}
$$

where we have defined $H_{t}(x):=\left(\rho_{t} * h^{p}\right)^{\frac{1}{p}}$.

Step 2. Fix $t_{0}>0$ and consider a cube $Q \subset \Omega$ such that its double $2 Q$ satisfies $\operatorname{dist}(2 Q, \partial \Omega)>t_{0}$. For a fixed $t \in\left(0, t_{0}\right)$ we write for notational simplicity $f, H$ instead of $f_{t}, H_{t}$ in the following and record, from Step 1, that for all dyadic rectangles $R \in \mathcal{R}(Q)$ the weak reverse Hölder inequality

$$
\left(f_{R} f^{p} \mathrm{~d} x\right)^{\frac{1}{p}} \leq K f_{2 R} f \mathrm{~d} x+\left(f_{2 R} H^{p} \mathrm{~d} x\right)^{\frac{1}{p}}
$$


holds. Denoting the maximal functions from Section 3 by $M=M_{\mathcal{R}(Q)}$ and $\tilde{M}=\tilde{M}_{\mathcal{R}(Q)}$, respectively, we deduce that $M\left[f^{p}\right]^{\frac{1}{p}} \leq K \tilde{M}[f]+\tilde{M}\left[H^{p}\right]^{\frac{1}{p}}$ on $Q$. For $q>p$ we estimate by elementary means

$$
\begin{aligned}
\text { LHS }:=\left(f_{Q} M\left[f^{p}\right]^{\frac{q}{p}} \mathrm{~d} x\right)^{\frac{1}{q}} & \leq\left(f_{Q}\left(K \tilde{M}[f]+\tilde{M}\left[H^{p}\right]^{\frac{1}{p}}\right)^{q} \mathrm{~d} x\right)^{\frac{1}{q}} \\
& \leq K\left(f_{Q} \tilde{M}[f]^{q} \mathrm{~d} x\right)^{\frac{1}{q}}+\left(f_{Q} \tilde{M}\left[H^{p}\right]^{\frac{q}{p}} \mathrm{~d} x\right)^{\frac{1}{q}}=: \text { RHS. }
\end{aligned}
$$

Here the left hand side is estimated using the lower bound from Proposition 3.1 and concavity of the function $s \mapsto s^{\frac{1}{p}}$, whereby

$$
\text { LHS } \geq\left(\frac{q}{2(q-p)}\right)^{\frac{1}{q}}\left[\left(f_{Q} f^{q} \mathrm{~d} x\right)^{\frac{1}{q}}-\left(f_{Q} f^{p} \mathrm{~d} x\right)^{\frac{1}{p}}\right],
$$

and the right hand side by Proposition 3.6 and again concavity of the function $s \mapsto s^{\frac{1}{p}}$ :

$$
\begin{aligned}
\text { RHS } \leq & K\left(\frac{q}{q-1}\right)^{2}\left(f_{Q} f^{q} \mathrm{~d} x\right)^{\frac{1}{q}}+K \frac{q}{q-1} 2^{2 n} f_{2 Q} f \mathrm{~d} x \\
& +\left(\frac{q}{q-p}\right)^{\frac{2}{p}}\left(f_{Q} H^{q} \mathrm{~d} x\right)^{\frac{1}{q}}+\left(\frac{q}{q-p}\right)^{\frac{1}{p}} 2^{\frac{2 n}{p}}\left(f_{2 Q} H^{p} \mathrm{~d} x\right)^{\frac{1}{p}} .
\end{aligned}
$$

At this stage we see that the exponent $q>p$ must be chosen so that

$$
\left(\frac{q}{2(q-p)}\right)^{\frac{1}{q}}>K\left(\frac{q}{q-1}\right)^{2}
$$

The choice $\left(\frac{q}{2(q-p)}\right)^{\frac{1}{q}}=2 K\left(\frac{q}{q-1}\right)^{2}$ leads to the range of exponents asserted in (1.2) and with this choice we can rearrange and simplify the above estimates to arrive at (with $q^{\prime}=q /(q-1)<p /(p-1)=$ $\left.p^{\prime}\right)$

$$
\begin{aligned}
\left(f_{Q} f^{q} \mathrm{~d} x\right)^{\frac{1}{q} \leq} & 2\left(f_{Q} f^{p} \mathrm{~d} x\right)^{\frac{1}{p}}+\frac{2^{2 n}}{q^{\prime}} f_{2 Q} f \mathrm{~d} x \\
& +2^{\frac{2 q+2-p}{p}}\left(K\left(q^{\prime}\right)^{2}\right)^{\frac{2 q-p}{p}}\left(f_{Q} H^{q} \mathrm{~d} x\right)^{\frac{1}{q}}+2^{\frac{q+1-p+2 n}{p}}\left(K\left(q^{\prime}\right)^{2}\right)^{\frac{q-p}{p}}\left(f_{2 Q} H^{p} \mathrm{~d} x\right)^{\frac{1}{p}} \\
\leq & 2^{2 n+1}\left(f_{2 Q} f^{p} \mathrm{~d} x\right)^{\frac{1}{p}}+4^{n+3}\left(p^{\prime}\right)^{6} K^{3}\left(f_{2 Q} H^{q} \mathrm{~d} x\right)^{\frac{1}{q}} \\
= & A\left(f_{2 Q} f^{p} \mathrm{~d} x\right)^{\frac{1}{p}}+B\left(f_{2 Q} H^{q} \mathrm{~d} x\right)^{\frac{1}{q}} .
\end{aligned}
$$

Now recall that $f=f_{t}, H=H_{t}$ and that we assume $f, h \in \mathrm{L}^{p}(\Omega)$. If $h \notin \mathrm{L}^{q}(2 Q)$, then there is nothing to prove so we may assume that $h \in \mathrm{L}^{q}(2 Q)$. Then we can pass to the limit $t \searrow 0$ in the penultimate inequality above and conclude with the desired bound.

4.1. Acknowledgments. This project was initiated at the Mittag-Leffler Institute during the special program "Evolutionary problems" in 2013. It was discussed and developed at the Oxford Mathematical Institute during Michaelmas Term 2015. BS would like to thank the institute for the hospitality and the nice environment. BS has been partially supported by the EPSRC Centre for Doctoral Training in PDEs and by a short term mobility grant at Federico II. JK gratefully acknowledges support from the Gnampa Visiting Professor program and thanks the Department of Mathematics at Federico II for kind hospitality during a number of visits. 


\section{REFERENCES}

[1] B. Bojarski, C. Sbordone and I. Wik: The Muckenhoupt class $A_{1}(\mathbb{R})$. Studia Math. 101 (1992), no. 2, 155-163.

[2] A. Cianchi and N. Fusco: Gradient regularity for minimizers under general growth conditions. J. Reine Angew. Math. 507 (1999), 15-36.

[3] R. Coifman and C. Fefferman: Weighted norm inequalities for maximal functions and singular integrals. Studia Math. 51 (1974), 241-250.

[4] D. Cruz-Uribe and C.J. Neugebauer: The structure of the reverse Hölder classes. Trans. Amer. Math. Soc. 347 (1995), no. 8 , 2941-2960.

[5] L. D'Apuzzo and C. Sbordone: Reverse Hölder inequalities: a sharp result. Rend. Mat. Appl. (7) 10 (1990), no. 2, 357-366.

[6] M. Dindoš and T. Wall: The sharp $A_{p}$ constant for weights in a reverse-Hölder class. Rev. Mat. Iberoam. 25 (2009), no. 2 , 559-594.

[7] J.L. Doob: Measure theory. Graduate Texts in Mathematics, 143. Springer-Verlag, New York, 1994. xii+210 pp.

[8] N. Fusco and C. Sbordone: Higher integrability of the gradient of minimizers of functionals with nonstandard growth conditions. Comm. Pure Appl. Math. 43 (1990), no. 5, 673-683.

[9] J. García-Cuerva and J.L.R. Francia: Weighted norm inequalities and related topics. North-Holland Mathematics Studies 116, North-Holland Publishing Co., Amsterdam 1985.

[10] F.W. Gehring: The $L^{p}$-integrability of the partial derivatives of a quasiconformal mapping. Acta Math. 130 (1973), 265277.

[11] M. Giaquinta and G. Modica: Regularity results for some classes of higher order nonlinear elliptic systems. J. Reine Angew. Math. 311/312 (1979), 145-169.

[12] E. Giusti: Direct methods in the calculus of variations. World Scientific Publishing Co., Inc., River Edge, NJ, 2003. viii+403 pp.

[13] T. Iwaniec: The Gehring lemma. Quasiconformal mappings and analysis (Ann Arbor, MI, 1995), 181-204, Springer, New York, 1998.

[14] T. Iwaniec and G. Martin: Geometric function theory and non-linear analysis. Oxford Mathematical Monographs. The Clarendon Press, Oxford University Press, New York, 2001. xvi+552.

[15] T. Kilpeläinen and P. Koskela: Global integrability of the gradients of solutions to partial differential equations. Nonlinear Anal. 23 (1994), no. 7, 899-909.

[16] J. Kinnunen: Sharp results on reverse Hölder inequalities. Ann. Acad. Sci. Fenn. Ser. A I Math. Dissertationes No. 95 (1994), $34 \mathrm{pp}$.

[17] J. Kinnunen: A stability result on Muckenhoupt's weights. Publ. Mat. 42 (1998), no. 1, 153-163.

[18] J. Kristensen and C. Melcher: Regularity in oscillatory nonlinear elliptic systems. Math. Z. 260 (2008), no. 4, 813-847.

[19] J. Kristensen and G. Mingione: The singular set of minima of integral functionals. Arch. Ration. Mech. Anal. 180 (2006), no. 3, 331-398.

[20] T. Kuusi, G. Mingione and Y. Sire: Nonlocal self-improving properties. Anal. PDE 8 (2015), no. 1, 57-114.

[21] T. Luque, C. Pérez and E. Rela: Reverse Hölder property for strong weights and general measures. J. Geom. Anal. 27 (2017), no. 1, 162-182.

[22] V.G. Maz'ya: Sobolev spaces with applications to elliptic partial differential equations. Second, revised and augmented edition. Grundlehren der Mathematischen Wissenschaften [Fundamental Principles of Mathematical Sciences], 342. Springer, Heidelberg, 2011. xxviii+866 pp.

[23] A. Melas: The Bellman functions of dyadic-like maximal operators and related inequalities. Adv. Math. 192 (2005), no. 2, $310-340$.

[24] A. Melas: A sharp $L^{p}$ inequality for dyadic $A_{1}$ weights in $\mathbb{R}^{n}$. Bull. London Math. Soc. 37 (2005), no. 6, 919-926.

[25] A. Melas, E. Nikolidakis and T. Stavropoulos: Sharp lower $L^{p}$-bounds for dyadic-like maximal operators. Proc. Amer. Math. Soc. 141 (2013), no. 9, 3171-3181.

[26] N.G. Meyers: An $L^{p}$-estimate for the gradient of solutions of second order elliptic divergence equations. Ann. Sc. Norm. Sup. Pisa 17 (1963), 189-206.

[27] N.G. Meyers and A. Elcrat: Some results on regularity for solutions of non-linear elliptic systems and quasi-regular functions. Duke Math. J. 42 (1975), 121-136.

[28] J. Martín and M. Milman: Gehring's lemma for nondoubling measures. Michigan Math. J. 47 (2000), no. 3, 559-573.

[29] B. Muckenhoupt: Weighted norm inequalities for the Hardy maximal function. Trans. Amer. Math. Soc. 165 (1972), 207226.

[30] A. Osekowski: Sharp inequalities for dyadic $A_{1}$ weights. Arch. Math. 101 (2013), 181-190.

[31] E.W. Stredulinsky: Higher integrability from reverse Hölder inequalities. Indiana Univ. Math. J. 29 (1980), no. 3, 407-413.

[32] V. Vasyunin: The sharp constant in the reverse Hölder inequality for Muckenhoupt weights. St. Petersburg Math. J. 15 (2004), 49-79.

Mathematical Institute

University of Oxford 
Andrew Wiles Building, Radcliffe Observatory Quarters Woodstock Road, Oxford OX2 6GG, United Kingdom

E-mail: kristens@maths.ox.ac.uk

Dipartimento di Matematica e Applicazioni

Università di Napoli Federico II

Via Cintia, 80126 Napoli, Italy.

E-mail: bstroffo@unina.it 